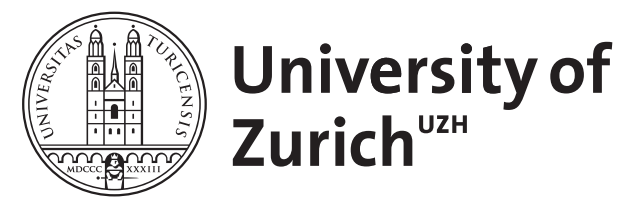

\title{
Der elektronische Mutterpass
}

\author{
Zimmermann, R ; Blöchlinger-Wegmann, B ; Kurmanavicius, J
}

\begin{abstract}
Zusammenfassung: Durch das Führen einer elektronischen Krankengeschichte für Schwangerschaft, Geburt und Wochenbett wurde es uns möglich, die elektronisch gespeicherten Daten im PDFFormat auf einen USB-Stick zu übertragen. In der Ambulanz der Klinik für Geburtshilfe am Universitätsspital Zürich erhalten seit Herbst 2005 alle Schwangeren einen mit Drittmitteln der Industrie finanzierten elektronischen Mutterpass. Er beinhaltet alle über die Schwangere gespeicherten Daten der Krankenakte einschließlich (Ultraschall-)Bildern. Der Stick wird nach jeder Kontrolluntersuchung neu synchronisiert. Die ersten Rückmeldungen der Schwangeren sind sehr positiv
\end{abstract}

DOI: https://doi.org/10.1007/s00129-006-1812-7

Posted at the Zurich Open Repository and Archive, University of Zurich ZORA URL: https://doi.org/10.5167/uzh-156720

Journal Article

Published Version

Originally published at:

Zimmermann, R; Blöchlinger-Wegmann, B; Kurmanavicius, J (2006). Der elektronische Mutterpass. Der Gynäkologe, 39(4):278-282.

DOI: https://doi.org/10.1007/s00129-006-1812-7 
Gynäkologe 2006 · 39:278-282

DOI 10.1007/s00129-006-1812-7

Online publiziert: 31. März 2006

๑) Springer Medizin Verlag 2006

Redaktion

K. Vetter, Berlin
R. Zimmermann · B. Blöchlinger-Wegmann - J. Kurmanavicius

Klinik für Geburtshilfe, Universitätsspital Zürich, Schweiz

Mutterpass

In Deutschland ist der Mutterpass seit 45 Jahren mit Erfolg in die klinische Praxis eingeführt. Die Verwendung eines Büchleins hat verschiedene Nachteile: Es ist unhandlich, benötigt eine doppelte Dokumentation, Handschriften sind teils schwer lesbar und bei Verlust ist eine aufwändige Kopie notwendig. Mit dem elektronischen Mutterpass in Form eines USB-Sticks können eine Reihe dieser Nachteile vermieden werden. Das hier vorgestellte Projekt kann als Vorstufe einer dezentralen Gesundheitskarte angesehen werden.

Das in Deutschland vor 45 Jahren eingeführte Mutterpassbüchlein ist im eigentlichen Sinne eine Erfolgsstory. Es hat die meisten seiner Ziele in beträchtlichem Maße erreicht. Durch die Definition, was zur Schwangerenvorsorge gehört, konnte eine weitgehende Standardisierung erzielt werden. Der Mutterpass erleichtert die Kommunikation zwischen ambulant tätigem Arzt und Klinik. Die Schwangere hat, wenn sie mit den Abkürzungen und Fachwörtern vertraut ist, einen Einblick in die eigene Krankenakte. In Notfällen hat der zugezogene Arzt sofort Einblick in die wichtigsten Untersuchungsbefunde. Und schließlich führt der Mutterpass zu einer Kostenersparnis durch Vermeidung von Doppeluntersuchungen. In Österreich ist der im Mutterpass dokumentierte Nachweis wahrgenommener Vorsorgeuntersuchungen Voraussetzung für die Gewährung von Mutterschafts- und Kindergeldleistungen [1].

Interessanterweise hat trotz offensichtlicher Erfolge die Idee des Mutterpasses außerhalb deutschsprachiger Länder wenig Verbreitung gefunden. Bereits in der
Schweiz konnte sich trotz guter nachbarschaftlicher Beispiele ein Mutterpass in den 26 regionalen Gesundheitssystemen nicht etablieren. Auch englischsprachige Literatur zu den Stichworten "maternity logbook“, „maternity records“ oder „maternity notes" fehlt weitgehend, und selbst in Großbritannien sind nur lokale Projekte umgesetzt worden (http://www.perinatal. nhs.uk/notes.htm). In einer Review in der Cochrane-Database vom 2004 konnten diesbezüglich insgesamt nur 3 randomisierte, kontrollierte Studien gefunden werden mit sehr kleinen Fallzahlen, zwei aus England und eine aus Australien [2]. Dies ändert jedoch nichts an der grundsätzlichen Nützlichkeit dieses Dokuments.

Der Mutterpass in Form eines kleinen Büchleins hat einige Nachteile, und zwar unabhängig von der medizinischpolitischen Diskussion, welche Tests zur normalen kostenfreien Schwangeren-vorsorge gehören und welche als individuelle Gesundheitsleistungen (IGeL) für die Schwangere zusätzlich abgerechnet werden.

Das Führen eines Mutterpasses in $\mathrm{Pa}$ pierform erfordert eine Doppeldokumentation mit dem ganzen damit verbundenen Zusatzaufwand. Der Mutterpass ist ein Unikat. Bei Verlust müssen die Daten mühsam aus der Krankenakte des Arztes kopiert werden. Im Internet findet man weitere Kritik wie „Leider wird er sehr oft nur unzureichend ausgefüllt" oder „Leider ist der Mutterpass ziemlich unpraktisch, was die Größe angeht. Er wurde von Männern gemacht, die keine Ahnung haben von den Problemen, die Handtaschen verursachen können."

Im Folgenden möchten wir ein Projekt mit einem elektronischen Mutterpass be- schreiben, das geeignet ist, einen Großteil dieser Nachteile zu eliminieren.

Die Klinik für Geburtshilfe am Universitätsspital Zürich ist das größte deutschsprachige Perinatalzentrum der Schweiz (2300 Entbindungen und etwa 800 pränatale stationäre Aufnahmen pro Jahr, große angegliederte Poliklinik). In der Ambulanz werden jährlich etwa 600 Schwangere während der ganzen und etwa weitere 300 während Teilen der Schwangerschaft betreut. Im Jahr 1994 wurde ein flächendeckendes Klinikinformationssystem (Perinat ${ }^{\circ}$ ) eingeführt, das im Sinne einer elektronischen Krankengeschichte die ambulante Schwangerenberatung, Ultraschall, Doppler, pränatale stationäre Dokumentation, Entbindungsdokumentation und Wochenbettsdokumentation beinhaltet [3]. In den Jahren 1999 und 2004 wurde das Systems wesentlich erweitert, eine der Innovationen machte mit weitgehender Parametrisierung der Daten die Einführung eines elektronischen Mutterpasses (EMP) möglich. Die Einführung eines EMPs bietet sich auch dank der in der Schweiz sehr hohen PC-Dichte an.

\section{Zielsetzung}

Das Projekt beabsichtigt sicherzustellen, dass jede Schwangere, die von uns betreut wird, jederzeit elektronisch über alle schwangerschaftsrelevanten Daten, Bilder, Broschüren, Notfallnummern etc. verfügt. Die Daten werden alle auf einen

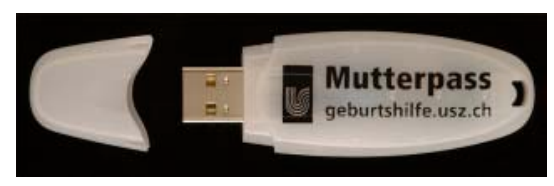

Abb. $1 \Delta$ Frontansicht des elektronischen Mutterpasses 


\section{Hier steht eine Anzeige.}

黛 Springer 


\section{Zum Thema}

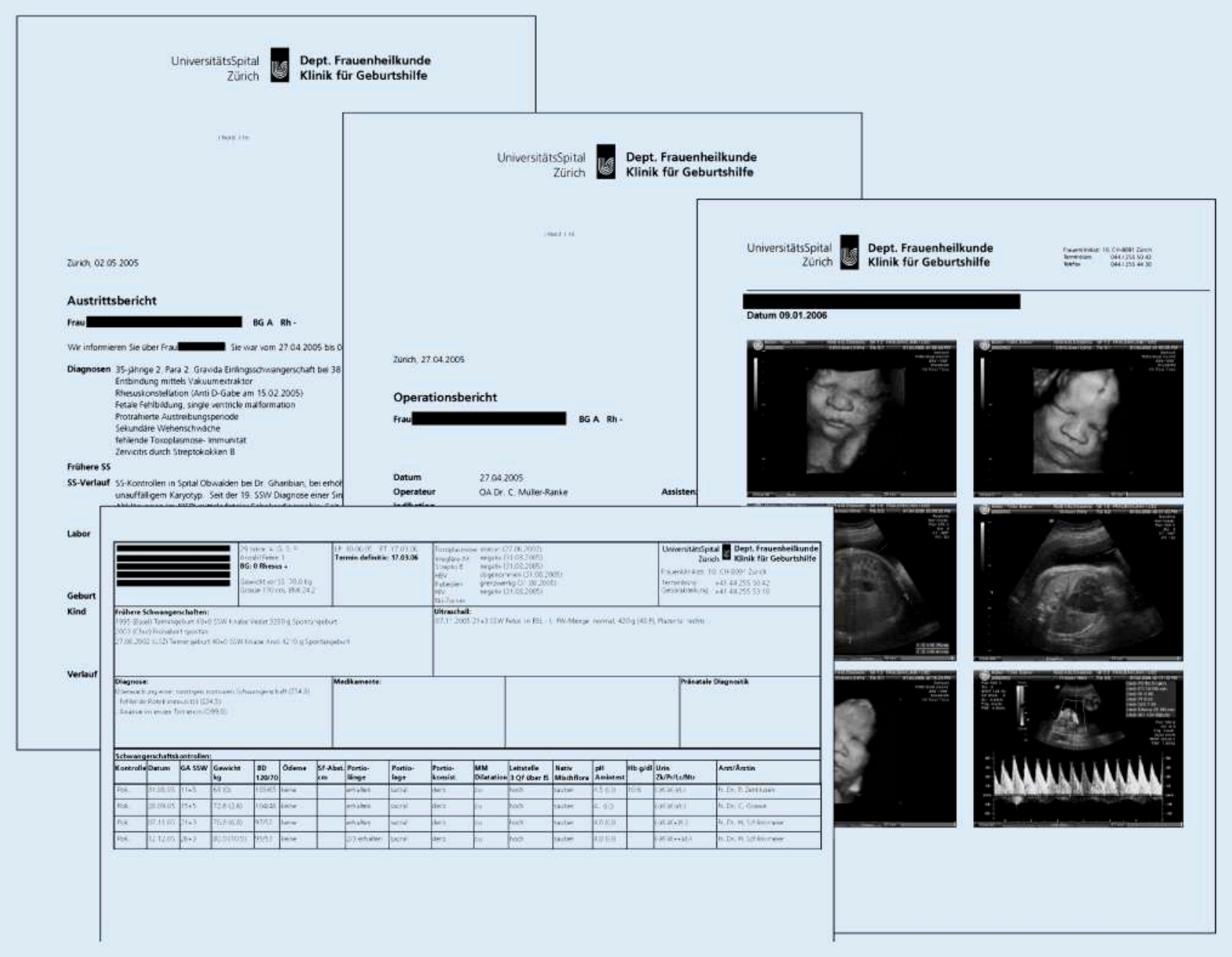

Abb. 2 A Auswahl von verschiedenen in einem PDF-File zusammengefassten Dokumenten aus der elektronischen Krankengeschichte

USB-Stick übertragen. Der Stick wird bei jeder Kontrolle neu synchronisiert.

\section{Details}

Anlässlich der ersten ambulanten Kontrolle wird der Schwangeren das Prinzip des elektronischen Mutterpasses durch den zuständigen Arzt mündlich erklärt. Zudem wird ein Informationsblatt zum EMP (in 8 verschiedenen Sprachen erhältlich) abgegeben.

Möchte die Schwangere eine elektronische Dokumentation, wird ein USBStick abgegeben. Bei jeder weiteren Kontrolle wird der Stick mit dem Patientinnenfile im Perinat synchronisiert. Dazu ist ein einziger Klick mit der Maus nötig, durch den die alten Daten komplett überschrieben werden. Bei diesem Vorgang wird kein File geöffnet, was die Virensicherheit erhöht.
Als Datenformat wurde Adobe PDF (Adobe Systems, San Jose/CA, USA) gewählt, damit plattformunabhängig alles gelesen werden kann. Als Inhalt war in einer ersten Phase vorgesehen:

- Anamneseblatt,

- Befundblatt Schwangerschaftskontrollen,

- Ultraschallbefunde,

- Ultraschallbilder (aus dem PACS),

- ggf. frühere Entlassungsberichte,

- ggf. frühere Operationsberichte,

- digitale Bilder (z. B. vom Neugeborenen),

- Schriftverkehr mit dem zuweisenden Arzt,

- klinikeigene Broschüre „Ratgeber für Schwangere",

- klinikeigene Broschüre „Ratgeber für Geburt und Wochenbett" und eine
- Informationsbroschüre des Staatssekretariats für Wirtschaft zum Thema Arbeitsrecht in der Schwangerschaft.

Das Projekt konnte mit einem vierwöchigen Programmieraufwand umgesetzt werden ( $\bullet$ Abb. 1). Die Finanzierung der Sticks erfolgte über die Industrie. Damit können lästige Depotgebühren von den Schwangeren vermieden werden. Als Gegenleistung dienen die USB-Sticks auf der Rückseite als Fläche für Werbebanner. Das Projekt ist kompatibel mit dem Patientengesetz des Kantons Zürich. Auch vom Datenschutzbeauftragten des Spitals wurde es - mit wenigen Auflagen - gutgeheißen. Die Auflagen betreffen zum einen den in das Informationsblatt aufzunehmenden Hinweis, dass bei der Benutzung des Sticks auf PCs oftmals temporäre Kopien gespeichert werden, die Fremde später lesen könnten. Die Schwangere 


\begin{tabular}{|c|c|c|}
\hline & Mutterpass aus Papier & Elektronischer Mutterpass \\
\hline \multirow[t]{8}{*}{ Pro } & \multirow[t]{8}{*}{ Ohne Hilfsmittel lesbar } & Einfacher Ersatz bei Verlust \\
\hline & & Handlich \\
\hline & & Enthält ganze Krankengeschichte \\
\hline & & Keine Doppeldokumentation \\
\hline & & Gut lesbar \\
\hline & & Vollständiger \\
\hline & & Kann zusätzliche Informationen enthalten \\
\hline & & Ermöglicht eine zentrale Schwangerschaftsdatenbank \\
\hline \multirow[t]{5}{*}{ Kontra } & Nur ausgewählte Information & Lesen nur am Computer möglich \\
\hline & Mäßig handlich & Erhöhter Zeitbedarf für die Dokumentation \\
\hline & Doppelte Dokumentation & Datenschutzfrage \\
\hline & Mäßige Lesbarkeit & Virensicherheit \\
\hline & Unikat & Lange Synchronisationszeit (z. Zt. noch) \\
\hline
\end{tabular}

wird aufgefordert, solche temporäre $\mathrm{Da}$ teien nach dem Lesen zu löschen. Außerdem wurde der Hinweis in das Informationsblatt aufgenommen, dass bei Verlust des Sticks der Finder alle medizinischen Daten lesen kann, weshalb der Stick immer so sicher wie ein Schlüssel oder eine Geldbörse aufbewahrt werden soll.

Da alle PCs unserer Klinik mit aktuellen Virenschutzprogrammen ausgerüstet sind, wurde die Gefahr einer unerwünschten Einschleppung von Viren als gering eingestuft. Auf jeden Fall ist das Risiko nicht höher als bei Verwendung von USB-Sticks zu anderen Zwecken.

- Auf einen Passwortschutz wurde bewusst verzichtet, um die Lesbarkeit in Notfällen nicht einzuschränken.

Nach der Synchronisation wird der Stick automatisch „ausgeworfen“, so dass auch weniger geübte Nutzer mit dem System gut klarkommen.

Als kleiner Nachteil des Systems muss die zur Zeit noch etwas lange Synchronisationszeit von gut 2 Minuten erwähnt werden. Der Zeitpunkt der Synchronisation muss gut in die Konsultation eingeplant werden, damit die Wartezeit nicht zu lange dauert.

In - Abb. 2 ist eine Auswahl von Dokumenten abgebildet, die auf dem Stick in einem einzigen PDF-File abgespeichert sind. Zentral ist das Übersichtsblatt über alle erfolgten Schwangerschaftsuntersuchungen, aber auch alte Entlassungs- oder Operationsberichte sind integriert.
Die Unterschiede zwischen der Papierform und der elektronischen Form eines Mutterpasses sind in $\bullet$ Tab. 1 zusammengefasst.

\section{Akzeptanz}

Der Stick wurde zunächst in einer einzelnen Sprechstunde über 4 Monate abgegeben. Durch die guten Rückmeldungen ermutigt erfolgte eine breitere Nutzung mit Abgabe in allen Sprechstunden ab Herbst 2005. In kurzer Zeit wurden über 400 EMPs verteilt. Neben der wahrgenommenen Sicherheit sind die Frauen auch äußerst zufrieden mit den mit abgespeicherten Ultraschallbildern.

\section{Ausblick}

Unser System. Aktuell wird das System im Rahmen einer Dissertation ausgewertet, um die Rückmeldungen von Schwangeren und Ärzten in die Weiterentwicklung einfließen zu lassen. Verbesserungen, die bereits in Planung sind, umfassen:

- Beschleunigung des Synchronisationsvorganges,

- Abspeichern eines Infofiles im Sinne eines „Schlüssels“ für das Verständnis der einzelnen Fachbegriffe und lip zur Präsentation unserer Klinik.

\section{Gesundheitskarte: Strategie zur Parti-}

zipation. In der Schweiz hat das Parlament im Jahr 2005 die Einführung einer nationalen Gesundheitskarte mit Hinweis auf die bislang ungelöste Datenschutzfra-
- Erweiterung um einen Multimediac-
Gynäkologe 2006 · 39:278-282

DOI 10.1007/s00129-006-1812-7

(c) Springer Medizin Verlag 2006

R. Zimmermann · B. Blöchlinger-Wegmann . J. Kurmanavicius

Der elektronische Mutterpass

Zusammenfassung

Durch das Führen einer elektronischen Krankengeschichte für Schwangerschaft, Geburt und Wochenbett wurde es uns möglich, die elektronisch gespeicherten Daten im PDFFormat auf einen USB-Stick zu übertragen. In der Ambulanz der Klinik für Geburtshilfe am Universitätsspital Zürich erhalten seit Herbst 2005 alle Schwangeren einen mit Drittmitteln der Industrie finanzierten elektronischen Mutterpass. Er beinhaltet alle über die Schwangere gespeicherten Daten der Krankenakte einschließlich (Ultraschall-)Bildern. Der Stick wird nach jeder Kontrolluntersuchung neu synchronisiert. Die ersten Rückmeldungen der Schwangeren sind sehr positiv.

Schlüsselwörter

Mutterpass · Elektronisch · USB-Stick

\section{The electronic maternity notes}

\section{Abstract}

The electronic storage of maternity notes, including information on pregnancy, delivery and the postpartum period, allows this information to be saved in PDF format on a USB stick. In the Obstetrics Clinic at the University Hospital of Zurich, all pregnant women receive their pregnancy notes on such a USB stick. This practice has been introduced in autumn 2005. The stick is industry sponsored. The stick contains all electronic hospital data, including ultrasound pictures, and is updated at each antenatal visit. The first feedback from the women involved has been very positive.

Keywords

Maternity notes - Electronic - USB stick 
ge vorläufig abgelehnt. Zur Abstimmung gestanden hatte ein Entwurf, ähnlich wie in anderen Ländern medizinische Daten einzelner Patienten zentral abzuspeichern und den Zugang über eine Gesundheitskarte zu ermöglichen.

\section{( ) Unser System erfordert prinzi- piell keine zentrale Datenbank}

Unser System erfordert prinzipiell keine zentrale Datenbank, alle damit verbundenen Datenschutzprobleme werden also vermieden, da die Originaldaten beim betreuenden Arzt abgespeichert bleiben und die Patientin lediglich eine elektronische Kopie davon besitzt. Es ist denkbar, dass verschiedene Ärzte und Kliniken ihre elektronischen Krankenakteneinträge auf ein und denselben USB-Stick abspeichern. Die Patientin hat so eine „Kopie“. Sie kann die Daten auch auf einem privaten PC abspeichern, um bei eventuellem Verlust ohne Kontakt mit allen Ärzten/ Kliniken wieder einen Stick aufbauen zu können. Mittlerweile sind USB-Sticks mit einer Kapazität von 10 GB im Handel, die wohl auch in Zukunft fast alle Wünsche hinsichtlich verfügbaren Speicherplatzes befriedigen können.

\section{Fazit für die Praxis}

Der EMP ist eine moderne und effiziente Alternative zur bekannten Papierversion. Er eliminiert eine Reihe von Nachteilen, die das Büchlein mit sich gebracht hat, ohne wesentliche neue Probleme zu schaffen. Als Modell für eine dezentrale Gesundheitskarte verlagert es die Datenschutzprobleme in die Selbstverantwortung des Patienten.

\section{Korrespondierender Autor \\ Prof. Dr. R. Zimmermann \\ Klinik für Geburtshilfe, Universitätsspital Zürich 8091 Zürich, Schweiz \\ roland.zimmermann@usz.ch}

Danksagung/Interessenkonflikt Folgenden Firmen danken wir für die Realisierung des beschriebenen Projekts: Ferring, Wallisellen; Biomed, Dübendorf; ZLB Behring, Bern; Bauerfeind, Oberrohrdorf und Robapharm, Allschwil. Die Präsentation des Themas ist unabhängig und die Darstellung der Inhalte produktneutral.

\section{Literatur}

1. Brezinka C (1998) Gesundheitsökonomie der Schwangerenbetreuung und Geburt in Österreich und den Niederlanden - ein Vergleich. Gynakol Geburtshilfliche Rundsch 38: 196-210

2. Brown HC, Smith HJ (2004) Giving women their own case notes to carry during pregnancy. The Cochrane Database of Systematic Reviews 24.02.2004

3. Kurmanavicius J, Burkhardt T, Zimmermann R (2004) EDV-unterstützte Dokumentation von UItraschalluntersuchungen. SchweizRundsch Med Praxis 93: 509-514

\section{Springer Medizin-Nachschlagewerke}

Elektronisch für unterwegs

Springer hat in den letzten Jahren kontinuierlich sein Publikationsangebot im Nachschlagebereich ausgebaut, insbesondere für den Bereich Medizin. Ab sofort sind fünf Springer Medizin-Nachschlagewerke elektronisch in einer PDA-Version (Portable Digital Assistant) verfügbar. Dazu gehören das Springer Lexikon Medizin sowie Der Große Reuter, das Springer Wörterbuch Medizin, das Springer Großwörterbuch Medizin und das Springer Taschenwörterbuch Medizin.

In der multimedialen PDA-Version des Lexikons und der Wörterbücher kann der Nutzer unterwegs jederzeit auf medizinische Fachbegriffe zugreifen. So umfasst z.B. das Springer Lexikon Medizin in der gedruckten Version auf 2.400 Seiten mehr als 80.000 Stichwörter, 2.800 farbige Abbildungen und Tabellen und wird ergänzt durch 44 ausführliche Essays zu wichtigen gesundheitlichen Fragen. All diese Informationen sind jetzt auch in der eBook-Version verfügbar.

Eine intuitive und nutzerfreundliche Bedienung garantiert eine komfortable Suche mit schnellen Suchergebnissen. Über eine Nachschlage-Funktion kann der Nutzer aus einem Werk heraus direkt in einem anderen eBook nachschlagen. Lesezeichen und persönliche Kommentare sind weitere Nutzungsmöglichkeiten.

Die neuen eBooks aus dem Springer Medizin Verlag laufen übrigens auf allen gängigen PDAs wie Palm und Pocket PC, auf Smartphones und dem PC. Sie sind im Internet unter www.med4mobile.de in einem auf medizinische Software für mobile Geräte spezialisierten Online-Shop zu kaufen. Weitere Informationen sind auch unter www.springer. com erhältlich. 